\title{
Effects of methane on giant planet's UV emissions and implications for the auroral characteristics
}

\author{
J. Gustin ${ }^{\mathrm{a}, *}$, J.-C. Gérard ${ }^{\mathrm{a}}$, D. Grodent ${ }^{\mathrm{a}}$, G.R. Gladstone ${ }^{\mathrm{b}}$, J.T. Clarke ${ }^{\mathrm{c}}$, W.R. Pryor ${ }^{\mathrm{d}}$, V. Dols ${ }^{\mathrm{e}}$, B. Bonfond ${ }^{\mathrm{a}}$, \\ A. Radioti ${ }^{\text {a }}$ L. Lamy ${ }^{\text {f }}$, J.M. Ajello ${ }^{\text {g }}$ \\ a Laboratoire de Physique Atmosphérique et Planétaire, Université de Liège, Liège, Belgium \\ b Southwest Research Institute, San Antonio, TX, USA \\ ${ }^{\mathrm{c}}$ Center for Space Physics, Boston University, MA, USA \\ ${ }^{\mathrm{d}}$ Central Arizona College, Coolidge, AZ, USA \\ e Laboratory for Atmospheric and Space Physics, Colorado University, Boulder, CO, USA \\ ${ }^{\mathrm{f}}$ Laboratoire d'Etudes Spatiales et d'Instrumentation en Astrophysique-Observatoire de Paris, Université Paris Diderot, Meudon, France \\ $\mathrm{g}$ Jet Propulsion Laboratory, California Institute of Technology, Pasadena, CA, USA
}

\section{A R T I C L E I N F O}

\section{Article history:}

Available online $\mathrm{xxxx}$

\section{Keywords:}

Ultraviolet

Giant planets

H2 spectroscopy

Methane

Aurora

Atmosphere

\begin{abstract}
A B S T R A C T
This study reviews methods used to determine important characteristics of giant planet's UV aurora (brightness, energy of the precipitating particles, altitude of the emission peak,...), based on the absorbing properties of methane and other hydrocarbons. Ultraviolet aurorae on giant planets are mostly caused by inelastic collisions between energetic magnetospheric electrons and the ambient atmospheric $\mathrm{H}_{2}$ molecules. The auroral emission is situated close to a hydrocarbon layer and may be attenuated by methane $\left(\mathrm{CH}_{4}\right)$, ethane $\left(\mathrm{C}_{2} \mathrm{H}_{6}\right)$ and acetylene $\left(\mathrm{C}_{2} \mathrm{H}_{2}\right)$ at selected wavelengths. As methane is the most abundant hydrocarbon, it is the main UV absorber and attenuates the auroral emission shorward of $1350 \AA$. The level of absorption is used to situate the altitude/pressure level of the aurora, hence the energy of the precipitated electrons, whose penetration depth is directly related to their mean energy. Several techniques are used to determine these characteristics, from the color ratio method which measures the level of absorption from the ratio between an absorbed and an unabsorbed portion of the observed auroral spectrum, to more realistic methods which combine theoretical distributions of the precipitating electrons with altitude dependent atmospheric models. The latter models are coupled with synthetic or laboratory $\mathrm{H}_{2}$ spectra and the simulated emergent spectra are compared to observations to determine the best auroral characteristics.

Although auroral characteristics may be very variable with time and locations, several typical properties may be highlighted from these methods: the Jovian aurora is the most powerful, with brightness around $120 \mathrm{kR}$ produced by electrons of mean energy $100 \mathrm{keV}$ and an emission situated near the $1 \mu \mathrm{bar}$ level ( $\sim 250 \mathrm{~km}$ above the 1 bar level) while Saturn's aurora is fainter ( $\sim 10 \mathrm{kR})$, produced by electrons less than $20 \mathrm{keV}$ and situated near the $0.2 \mu$ bar level $(\sim 1100 \mathrm{~km})$.
\end{abstract}

(c) 2013 Elsevier Inc. All rights reserved.

\section{Introduction}

The atmosphere of the giant planets is composed for the most part of hydrogen (atomic $\mathrm{H}$ and molecular $\mathrm{H}_{2}$ ), helium $(\mathrm{He})$ and methane $\left(\mathrm{CH}_{4}\right)$. Methane is produced in the deep atmosphere by pyrolysis and is transported upwards by diffusion or convection into the stratosphere, where its photolysis produces hydrocarbons like ethane $\left(\mathrm{C}_{2} \mathrm{H}_{6}\right)$ and acetylene $\left(\mathrm{C}_{2} \mathrm{H}_{2}\right)$. These products are transported by eddy mixing down into the troposphere where they undergo thermochemical reactions back to methane.

\footnotetext{
* Corresponding author.

E-mail address: gustin@astro.ulg.ac.be (J. Gustin).
}

The hydrocarbons are important sources and/or sinks of heat and thus play a major role in the dynamics and structure of the upper atmosphere. They also have a significant absorption crosssection in the ultraviolet (UV) spectral window (Extreme UV in

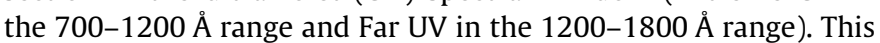
property is used in auroral studies to determine the altitude of the peak emission and the characteristics of precipitating auroral particles.

The UV aurora on giant planets were first detected by the Voyager missions [5] for Jupiter, Broadfoot et al. [6] for Saturn, Broadfoot et al. [7] for Uranus, Broadfoot et al. [58] for Neptune). These emissions appear as "ovals" and discrete emissions located either closer or further from the poles in the case of Jupiter and Saturn, as the result of complex interactions between the solar wind, the 
magnetosphere and the ionosphere. The satellites also play a key role, as they are a major source of charged particles in the vicinity of these planets (Io for Jupiter and Enceladus for Saturn). A thorough review of the auroral processes may be found in Clarke et al. [9] for Jupiter and Kurth et al. [38] for Saturn.

Uranus is a more intriguing case owing to its highly inclined rotational axis $\left(98^{\circ}\right)$ combined with dipole tilt of $-60^{\circ}$ [47]. According to the very few available observations, its aurora consists of northern and southern ring-like emissions plus localized bright spots [40].

Aurora at Neptune, detected on the nightside, appear as two types: (1) emission extending from $55^{\circ} \mathrm{S}$ to $50^{\circ} \mathrm{N}$ confined over longitudes $0^{\circ}$ to $60^{\circ} \mathrm{W}$ and (2) mostly southern emissions at longitudes $180^{\circ}-270^{\circ} \mathrm{W}$. This configuration is probably due to the dipole tilt of $47^{\circ}$ with the rotational axis. A comprehensive overview of giant planet auroras may be found in Bhardwaj and Gladstone [3].

This manuscript describes the techniques used to study the interaction between auroral emission and hydrocarbons and determine important characteristics of this magnetosphere-ionosphere coupling. The results presented focus on Jupiter and Saturn studies. The case of Uranus is not developed because of the lack of high quality spectral observations, needed to perform the procedures presented here.

\section{Auroral UV spectroscopy}

Comparisons between observed auroral spectra and models reveal that the auroral emission observed in the UV range is mainly caused by inelastic collisions between atmospheric $\mathrm{H}_{2}$ molecules and electrons of magnetospheric origin precipitating on top of the atmosphere (called primary electrons $e_{p}$ ). These primary electrons ionize $\mathrm{H}_{2}$, initially in the electronic ground-state level $\left(\mathrm{X}^{1} \Sigma_{\mathrm{g}}^{+}\right)$, and create secondary electrons $e_{s}$ :

$e_{p}+\mathrm{H}_{2} \rightarrow \mathrm{H}_{2}^{+}+e_{s}+e_{p}$

The primary and secondary electrons interact with $\mathrm{H}_{2}$ following the probability given by the various cross-sections (ionizations, electronic excitations, vibrational and rotational excitations). Above $50 \mathrm{eV}$, the ionization and the electronic excitation cross-sections have comparable behavior with respect to the electron energy, but ionization is from $\sim 5$ to $\sim 50$ times more probable (depending on the electronic level considered). Primary and secondary electrons are thus able to ionize other $\mathrm{H}_{2}$ molecules following (1) but can also excite them to upper electronic levels $\mathrm{B}^{1} \Sigma_{\mathrm{g}}^{+}, \mathrm{C}^{1} \prod_{\mathrm{u}}, \mathrm{B}^{\prime 1} \Sigma_{\mathrm{u}}^{+}, \mathrm{D}^{1} \prod_{\mathrm{u}}, \mathrm{B}^{\prime \prime 1} \Sigma_{\mathrm{u}}^{+}, \mathrm{D}^{\prime 1} \prod_{\mathrm{u}}$ :

$e_{x}+\mathrm{H}_{2} \rightarrow \mathrm{H}_{2}^{*}\left(\mathrm{~B}, \mathrm{C}, \mathrm{B}^{\prime}, \mathrm{D}, \mathrm{B}^{\prime \prime}, \mathrm{D}^{\prime}\right)+e_{x}$

where $x$ refers to $p$ (primary) or $s$ (secondary) electrons.

The bulk of the UV emission in 700-1800 $\AA$ is caused by reaction (2), which initiate de-excitation of excited $\mathrm{H}_{2}^{*}$ through the $\mathrm{B} \rightarrow \mathrm{X}, \mathrm{C} \rightarrow \mathrm{X}, \mathrm{B}^{\prime} \rightarrow \mathrm{X}, \mathrm{D} \rightarrow \mathrm{X}, \mathrm{B}^{\prime \prime} \rightarrow \mathrm{X}, \mathrm{D}^{\prime} \rightarrow \mathrm{X}$ rovibronic (rotational-vibrational-electronic) transitions and corresponding $\mathrm{H}_{2}$ continua and produces UV photons. The energy degradation process of primary and secondary electrons (i.e. losing their kinetic energy by ionizing $\mathrm{H}_{2}$ and creating other secondary electrons) continues until they are thermalized in the ambient atmosphere. Fig. 1 shows the contribution of each system band to the total $\mathrm{H}_{2}$ UV emission, obtained from a synthetic spectral model developed by Gustin et al. [27]. When de-excitation occurs at internuclear distance far enough from the minimum of the $\mathrm{H}_{2}$ potential energy, transitions connect to the dissociation continuum of $\mathrm{X}^{1} \Sigma_{\mathrm{g}}^{+}$state and form $\mathrm{H}(1 \mathrm{~s})$ atoms. Since the kinetic energy of these atoms is not discrete, the emitted photons produce a continuum emission. This component is mainly due to $\mathrm{B} \rightarrow \mathrm{X}$ transitions and dominates the FUV $\mathrm{H}_{2}$ spectrum between 1550 and $1650 \AA$, as illustrated in Fig. 1. In addition to these direct excitations (where the populating process of the excited electronic states is due to collisions and is assumed to follow the same rules as radiative excitation), the $\mathrm{B}^{1} \Sigma_{\mathrm{g}}^{+}$ state can also be populated through the optically forbidden component $\mathrm{X} ;{ }^{1} \Sigma_{\mathrm{g}}^{+} \rightarrow \mathrm{E}, \mathrm{F}^{1} \Sigma_{\mathrm{g}}^{+}$followed by the cascade transition $\mathrm{E}, \mathrm{F} ;{ }^{1} \Sigma_{\mathrm{g}}^{+} \rightarrow \mathrm{B} ;{ }^{1} \Sigma_{\mathrm{g}}^{+}$. While the excitation cross-section of the $\mathrm{B}$ and $C$ states is maximum near electron energy of $38 \mathrm{eV}$, the $\mathrm{E}, \mathrm{F}$ excitation cross-section peaks at $\sim 18 \mathrm{eV}$. The effect of the cascade process enhances emission around $1350 \AA$ A. Other cascade transitions through the $\mathrm{GK}, \mathrm{HH}, \ldots$. electronic states exist but have a minor contribution. The UV emission is dominated by the Lyman $(\mathrm{B} \rightarrow \mathrm{X})$ and Werner $(\mathrm{C} \rightarrow \mathrm{X})$ systems and associated continua, as they contribute $\sim 90 \%$ of the total $\mathrm{H}_{2}$ emission ([32]).

The other major features observed in the UV aurora come from dissociative excitation:

$e_{s}+\mathrm{H}_{2} \rightarrow \mathrm{H}+\mathrm{H}+e_{s}+h v$,

where $h v$ is a photon from the atomic hydrogen Lyman series. Although the Lyman lines may originate from scattering of solar Lyman lines or direct excitation of $\mathrm{H}$ atoms, dissociative excitation dominates the production of the auroral Lyman lines and contributes $\sim 99 \%$ [25].

\section{Effects of methane on UV emissions}

A hydrocarbon layer interacts with the auroral photons as they escape the atmosphere and attenuates the emission in specific wavelength ranges. The hydrocarbon absorption cross-sections combine photoabsorption, photoionisation and neutral dissociation processes, whose efficiency depends on the energy of the incident photon. Although most hydrocarbons are effective UV absorbers, only the most abundant $\mathrm{CH}_{4}, \mathrm{C}_{2} \mathrm{H}_{6}$ and $\mathrm{C}_{2} \mathrm{H}_{2}$ are usually considered in auroral studies. Fig. 2a shows the ultraviolet absorption cross-section of methane, ethane and acetylene. Measurements reported in several references are joined together in order to cover the full UV bandwidth. Although the most recent works are generally employed, some older ones are still necessary to smoothly merge data from different studies. In the case of methane for example, absorption cross-section at $1175 \AA$ varies from $2.45 \times 10^{-17} \mathrm{~cm}^{2}$ in Laufer and McNesby [39] to $1.70 \times 10^{-17} \mathrm{~cm}^{2}$ in Ditchburn [12], with several other references in between these values. While the most recent works appear as the adequate choice, as they use more precise techniques and generally provide higher spectral resolution, they may look inconsistent compared to older references, especially when a merging between different curves has to be done to cover the whole UV bandwidth. This illustrates well the difficulty to select the best appropriate curves, and the (relative) uncertainty brought by cross-section deteminations.

Although $\mathrm{CH}_{4}, \mathrm{C}_{2} \mathrm{H}_{6}$ and $\mathrm{C}_{2} \mathrm{H}_{2}$ cross-sections are of comparable magnitude, methane is much more abundant in giant planets' atmospheres than the other hydrocarbons and consequently, the optical depth related to the auroral emission attenuation is dominated by methane. The vertical optical depth, i.e. the absorption cross-section multiplied by the vertical column density of the corresponding hydrocarbon, is illustrated in Fig. $2 \mathrm{~b}$ for a pressure level of $2 \mu$ bar in Jupiter's atmosphere, where acetylene and ethane have column abundances 50 and 12.5 times lower than methane, respectively. The column densities are inferred from a model of the Jovian atmosphere relating the relative amount of each hydrocarbon ([45]). It is seen that the absorption is dominated by methane shortward of $1350 \AA$. The attenuation is negligible longward of $1350 \AA$, except for the moderate signature of ethane between 1350 and $1400 \AA$ and acetylene at 1480 and $1520 \AA$. Acetylene signatures are illustrated in Fig. $4 \mathrm{~b}$ for a pressure level of $3 \mu \mathrm{bar}$ at Jupi- 


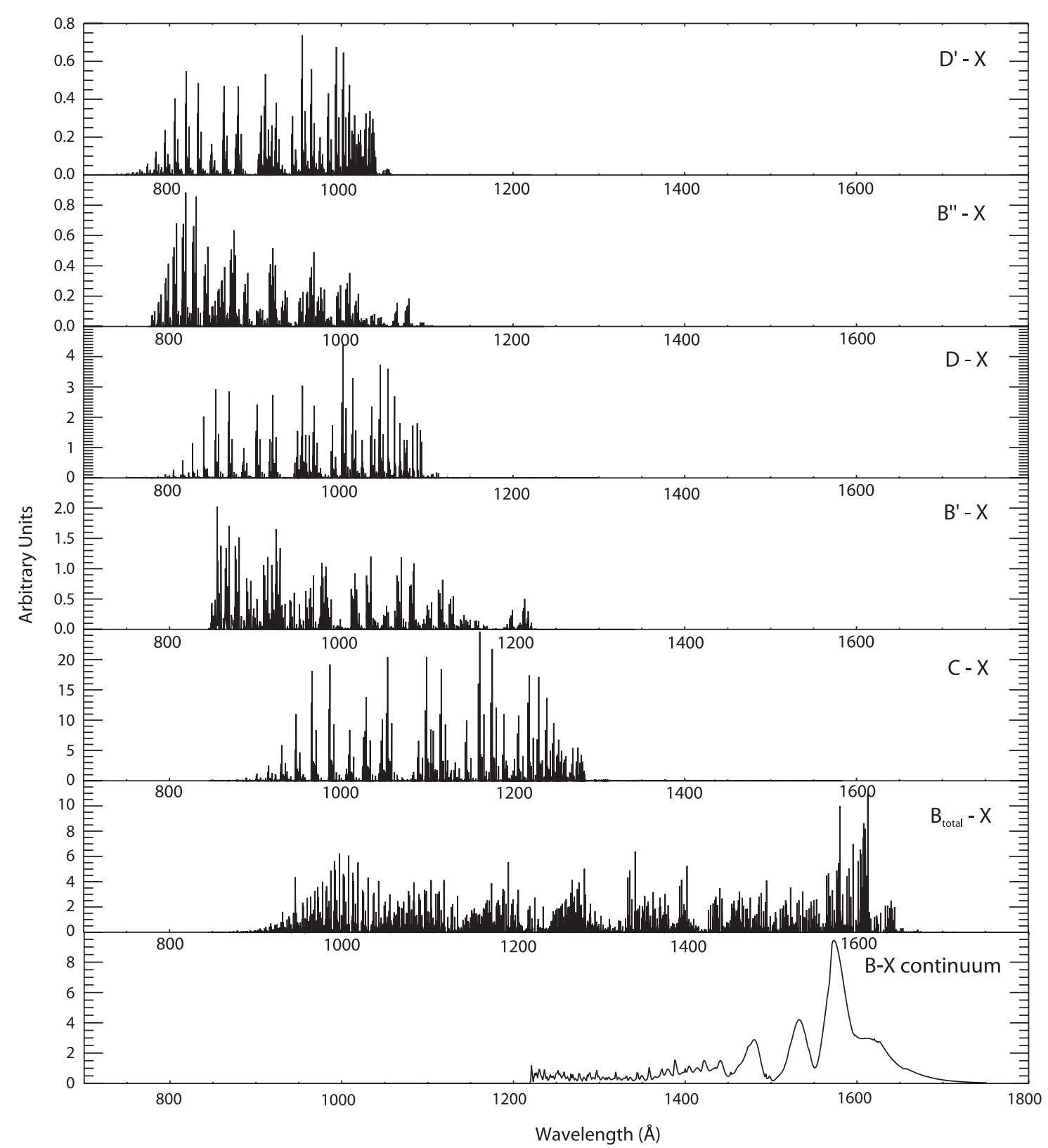

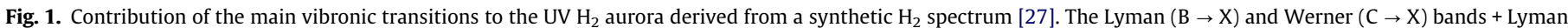
continuum dominate the signal and contribute $\sim 90 \%$ of the total emission.

ter. As a matter of fact, mentions to "hydrocarbons" will refer to "methane" in the following sections, unless otherwise stated. While acetylene has a limited effect on UV auroral absorption, it is the main contributor to the reflected sunlight attenuation and modulates this emission longward of $1550 \AA$.

As described by Eqs. (1) and (2), primary electrons precipitate on top of the atmosphere and lose their energy as they ionize, dissociate, excite $\mathrm{H}_{2}$ and create less energetic secondary electrons. Primaries may be modeled by a mono-energetic beam or more complex distributions such as Kappa or Maxwellian functions. This energy degradation process is illustrated in Fig. 3a in the case of Jupiter. It shows the energy spectrum of an incident flux of electrons composed of three Maxwellian distributions at the top of the atmosphere [25]. The shape of this distribution changes as the electrons penetrate deeper in the atmosphere: the electron population increases at low energies and decreases at the same time for the higher energies. The top layers of the atmosphere con- tribute insignificantly to the auroral emission because of the low $\mathrm{H}_{2}$ density and low electronic excitation cross-section for high electron energies. As the electron beam reaches deeper layers, more electrons with energies in the range $\sim 15$ to $\sim 300 \mathrm{eV}$ (i.e. where $\mathrm{H}_{2}$ ionization and electronic excitation cross-sections are maximum) are created and interact with denser layers of $\mathrm{H}_{2}$. The resulting auroral volume emission rate (VER) is illustrated in Fig. 3b from the two-stream model by Grodent et al. [25], briefly explained later in this section. In this model, the VER is maximum near the $1 \mu$ bar level, corresponding to an altitude of $\sim 250 \mathrm{~km}$, as constrained by Galileo visible observations [54]. In giant planet studies, it is generally assumed that $0 \mathrm{~km}$ corresponds to the 1 bar level. The energy of the precipitating particles thus reaches several $\mathrm{keV}$ or tens of $\mathrm{keV}$, while the energy of the electrons effectively producing the aurora are more in the $15-300 \mathrm{eV}$ range.

The emitted photons interact with the hydrocarbon layer situated near the VER and may experience absorption. A Beer-Lambert 

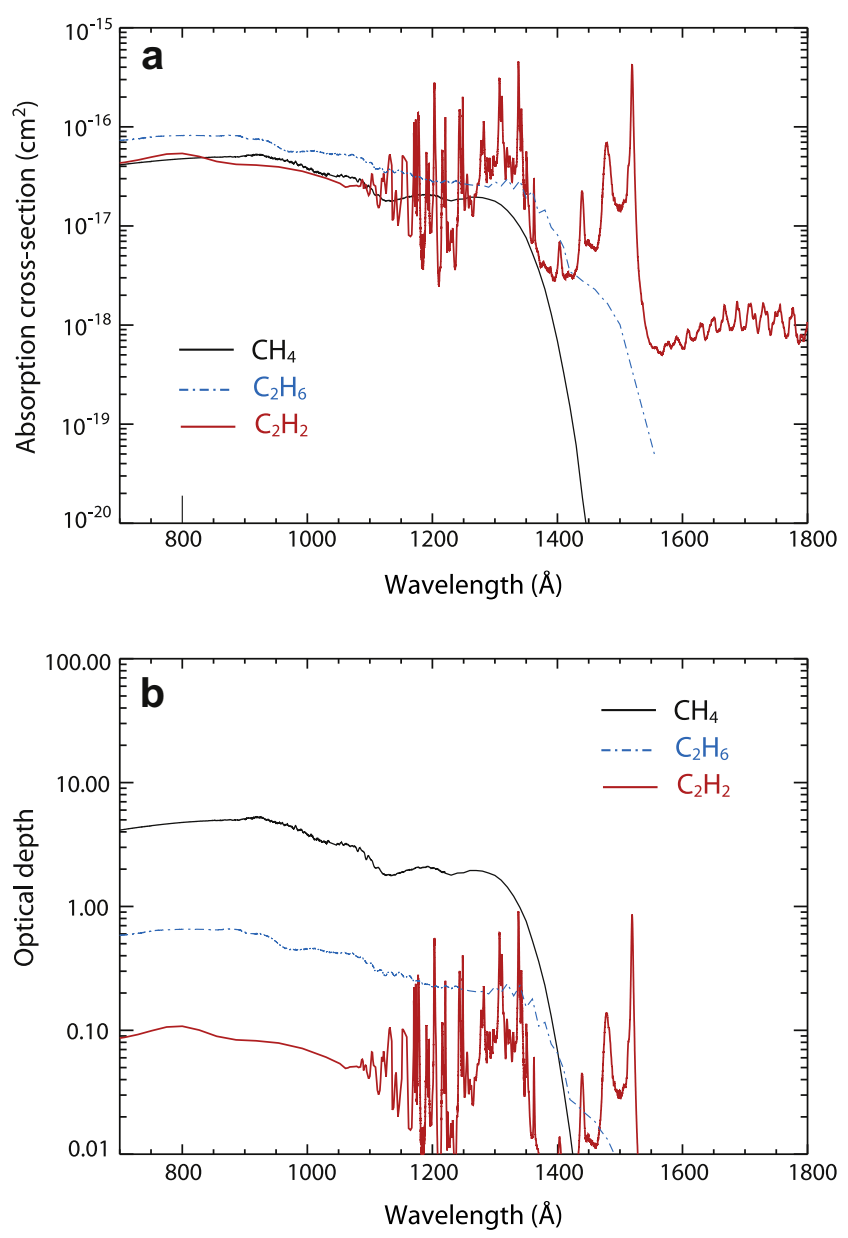

Fig. 2. (a) UV absorption cross-section for the three main hydrocarbons observed on giant planets. The methane cross-section comes from Au et al. [1] in 10 a $520 \AA$, Kameta et al. [34] in 520 a $1220 \AA$, and Lee et al. [41] in 1220 a $1520 \AA$ range. Ethane cross-section uses Kameta et al. [35] from 138 to $1246 \AA$ and Chen and $\mathrm{Wu}$ [8] from 1280 to $1600 \AA$. Acetylene uses Cooper et al. [11] from 62 to $1052 \AA$, Nakayama and Watanabe [46] from 1052 to $1170 \AA$ and $W u$ et al. [56] from 1170 to $2292 \AA$. Acetylene exhibits moderate cross-section above $1550 \AA$, which has no effect on aurora but has significant effect on reflected sunlight emission longward of $1550 \AA$. (b) Relative optical depth, i.e. product between the cross-section displayed in (a) and the column density in the case of the Jovian atmosphere.

law is used to relate the intrinsic auroral (unabsorbed) radiated emission at a specific wavelength $I_{\lambda}^{\text {unabs }}$ to the observed (absorbed) emission $I_{\lambda}^{\text {abs }}$, with $\tau_{\lambda}$ being the sum of individual optical depth due to hydrocarbons along the ray path:

$I_{\lambda}^{\mathrm{abs}}=I_{\lambda}^{\mathrm{unabs}} e^{-\tau}$

Fig. $3 \mathrm{~b}$ also shows the vertical variation of methane optical depth at $1250 \AA$, i.e. the methane column density profile multiplied by the absorption cross-section at $1250 \AA$. The optical depth reaches unity near $250 \mathrm{~km}$ and auroral photons shortward of $1350 \AA$ are significantly absorbed below this altitude, while most photons emitted upwards of $250 \mathrm{~km}$ escape the atmosphere. Fig. 4a illustrates the effect of absorption on auroral spectra. The blue curve is a laboratory spectrum obtained from impact of $100 \mathrm{eV}$ electrons on $\mathrm{H}_{2}$ gas at $300 \mathrm{~K}$ [15], ideal to simulate the intrinsic auroral emission. The observed Lyman alpha line in this spectrum is the result of collisions following Eq. (3). Absorption by the three main hydrocarbons has been applied to this laboratory spectrum by using the modeled Jovian atmosphere of Moses et al. [45]. Major attenuation below $1350 \AA$ is clearly observed on the green curve simulating the observed emergent emission. A linear plot of $\mathrm{CH}_{4}$ cross-section is also provided and clearly demonstrates the effect of methane UV absorption. A weak attenuation by $\mathrm{C}_{2} \mathrm{H}_{2}$ is also noticed in this case, at $\sim 1480$ and $\sim 1520 \AA$. Because of a very similar cross-section shape, it is difficult to discriminate between $\mathrm{CH}_{4}$ and $\mathrm{C}_{2} \mathrm{H}_{6}$ absorption, but $\mathrm{CH}_{4}$ is always assumed to be the main contributor to the brightness reduction below $1350 \AA$ because of its larger abundance compared to other hydrocarbons. Attenuation specific to ethane is however occasionally noticed in observations around the $1450 \AA$ region (an obvious example is illustrated in Figs. 9 and 10 of Dols et al. [13]). It should be noted that spectra displayed in Fig. 4 may be considered as ideal cases: observations with moderate signal to noise ratio sometimes makes it difficult to point out all the hydrocarbon signatures and determine their abundances with high confidence.

Since the level of absorption is linked to the altitude of emission, it is an indicator of the mean energy $\langle E\rangle$ of the precipitating electrons. Three methods are used to determine $\langle\mathrm{E}\rangle$, all making use of FUV spectra for two reasons. First, as the long wavelength portion of the spectrum is not affected by methane, it is used as a reference to determine the quantity of absorbing molecules in the short wavelength part of the FUV emission. Second, most space-born UV spectrographs operate in the FUV bandwidth, which allows observation of stellar objects without being affected by EUV interstellar absorptions. The majority of auroral UV observations thus involve the FUV spectral window.

The first method used to establish the auroral characteristics considers the aurora as an emitting layer, overlaid by an absorbing layer of hydrocarbons (e.g. Gustin et al. [30]). A fitting technique which compares the observed UV spectrum and attenuated laboratory (or synthetic) spectra following Eq. (4) is performed until the minimum chi-square is reached. The altitude and pressure level associated with the hydrocarbon column giving the best fit are then obtained from an atmospheric model which, in turn, provides the $\mathrm{H}_{2}$ column overlying the auroral emission. A stopping power table giving the average path length traveled by mono-energetic electrons into $\mathrm{H}_{2}$ as they slow down to rest, or an electron transport code may then be used to estimate $\langle E\rangle$. A table of stopping power may be found in the ESTAR database http://physics.nist.gov/PhysRefData/Star/Text/ESTAR.html. As the absorption may be very different from one case to another, Eq. (4) is also used to derive the intrinsic brightness of the aurora once the level of attenuation is determined. This is required to investigate the intrinsic temporal or spatial variation of auroral features and connect them to all mechanisms involved in the auroral production, such as acceleration processes, variations of magnetic field and solar winds interactions.

A second, more realistic technique used to establish the auroral characteristics employs a complete one-dimentional model of the atmosphere that interacts with an incident electron beam as explained above and in Fig. 3. The initial atmospheric model is perturbed by the auroral energy input and adjusts its characteristics following the continuity equation for the electron flux, the heat conservation equation for the temperature profile, and the molecular and turbulent diffusions for the major atmospheric constituents [25]. The final atmosphere is divided into several layers and coupled with a synthetic spectral generator which uses (1) the local temperature to distribute the vibrational and rotational population of the $\mathrm{H}_{2}$ molecules in $\mathrm{X}^{1} \Sigma_{\mathrm{g}}^{+}$, (2) the electron energy distribution to excite $\mathrm{H}_{2}$ and populate the excited electronic levels, (3) the local $\mathrm{H}_{2}$ density to weight the contribution of each layer to the total emergent spectrum, and (4) the $\mathrm{CH}_{4}, \mathrm{C}_{2} \mathrm{H}_{2}$ and $\mathrm{C}_{2} \mathrm{H}_{6}$ profiles to absorb the emission at selected wavelengths. The emergent spectrum, consisting of the sum of all the individual spectra, is compared to the observations. Several electron distributions are tested with a chi-squared minimization procedure until a good match between the observed and synthetic spectra is obtained. 

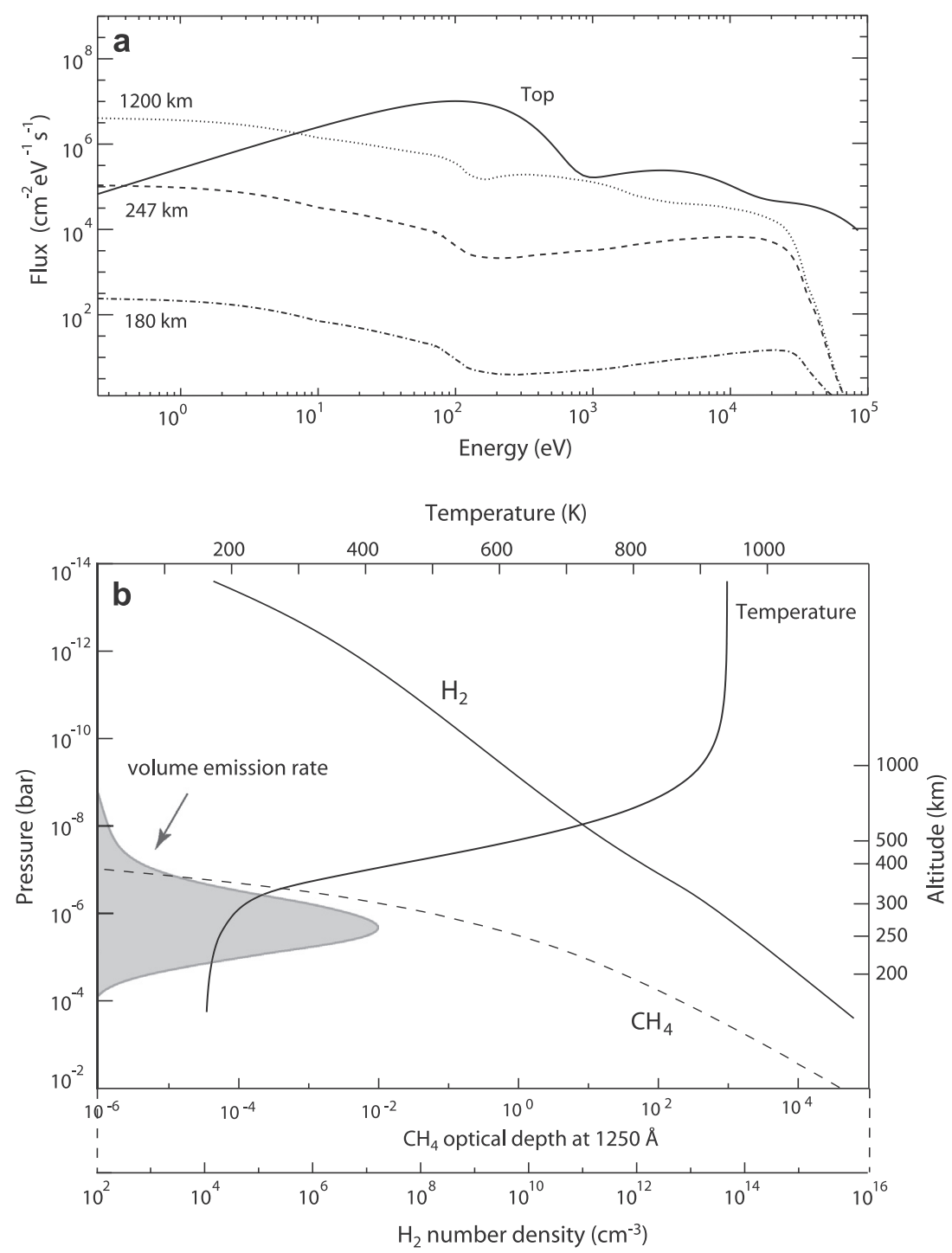

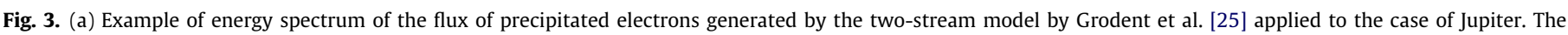
distribution on top of the atmosphere illustrated here is the combination of three Maxwellian distributions. As these primary electrons penetrate into the atmosphere, they create secondary electrons and the shape of the distribution evolves toward more low energetic electrons. (b) Jovian auroral atmosphere from the Grodent et al. [25] model associated with the electron precipitation described in a). The volume emission rate of auroral photons is the optimization of secondary electron interactions with $\mathrm{H}_{2}$ molecules. The linear volume emission rate shown peaks at $\sim 16 \times 10^{3}$ photons $\mathrm{cm}^{-3} \mathrm{~s}^{-1}$ for the whole UV range. The altitude profile of methane optical depth at $1250 \AA \AA$ in dashed line shows that the auroral photons are mostly affected by absorption below the $1 \mu$ bar level.

The electron distribution providing the best fit characterizes the energy distribution, mean energy and flux of the primary precipitating electrons. It should be noted that several studies (Trafton et al. [53] and Liu and Schultz [43] for example) indicate that the UV aurora is principally due to electron precipitation. Indeed, precipitation of each proton or ion on top of the atmosphere creates hundred of secondary electrons which mask the signature of the original precipitating particle. As explained by Waite et al. [55] or Kharchenko et al. [36], ion precipitation (principally due to sulfur and oxygen ions) is a minor contributor to the UV aurora but is the main source of the X-ray aurora through charge exchange (e.g. Branduardi-Raymont et al. [4], Hui et al. [33]).

For historical reasons, a third, simpler technique without spectral comparison is also often considered. It uses the color ratio $C R=I(1550-1620 \AA) / I(1230-1300 \AA)$ with I the auroral intensity in photon units [57], which is a quantitative measure of the absorption. The CR is $\sim 1.1$ for an unattenuated emergent spectrum and can reach values above 10 for strongly absorbed auroral spectra. An emergent spectrum with $C R=2.5$, typical value for Jovian aurora, is illustrated in Fig. 4a. To determine $\langle E\rangle$ from the CR, simulations with both the mono and multilayer models must be performed beforehand to produce a relationship between $\langle E\rangle$ and $\mathrm{CR}$ (see for example Fig. 4 of Gérard et al. [17]). The observed CR is calculated from the data and $\langle E\rangle$ is directly inferred from the CR-energy relationship. The color ratio method has been used in slightly different form in the litterature, e.g. Livengood et al. [44] or Rego et al. [51].

An aspect that must also be considered in all these approaches is the geometry of the observations. The auroral regions under study are rarely seen from nadir, and the emission angle $\chi$ (angle between the vector normal to the atmosphere and the instrument pointing vector) must be taken into account in these procedures. It is generally assumed that the observed intensity and hydrocarbon column vary as the secant of the emission angle $\chi$, which is a good approximation for angles less than $75^{\circ}$ in a spherical atmosphere, but the Chapman function $\mathrm{Ch}(\chi)$ is preferred for larger angles ([24]). Therefore a given observed CR or methane column does not correspond to a fixed value of the altitude and $\langle E\rangle$, as $\chi$ must 

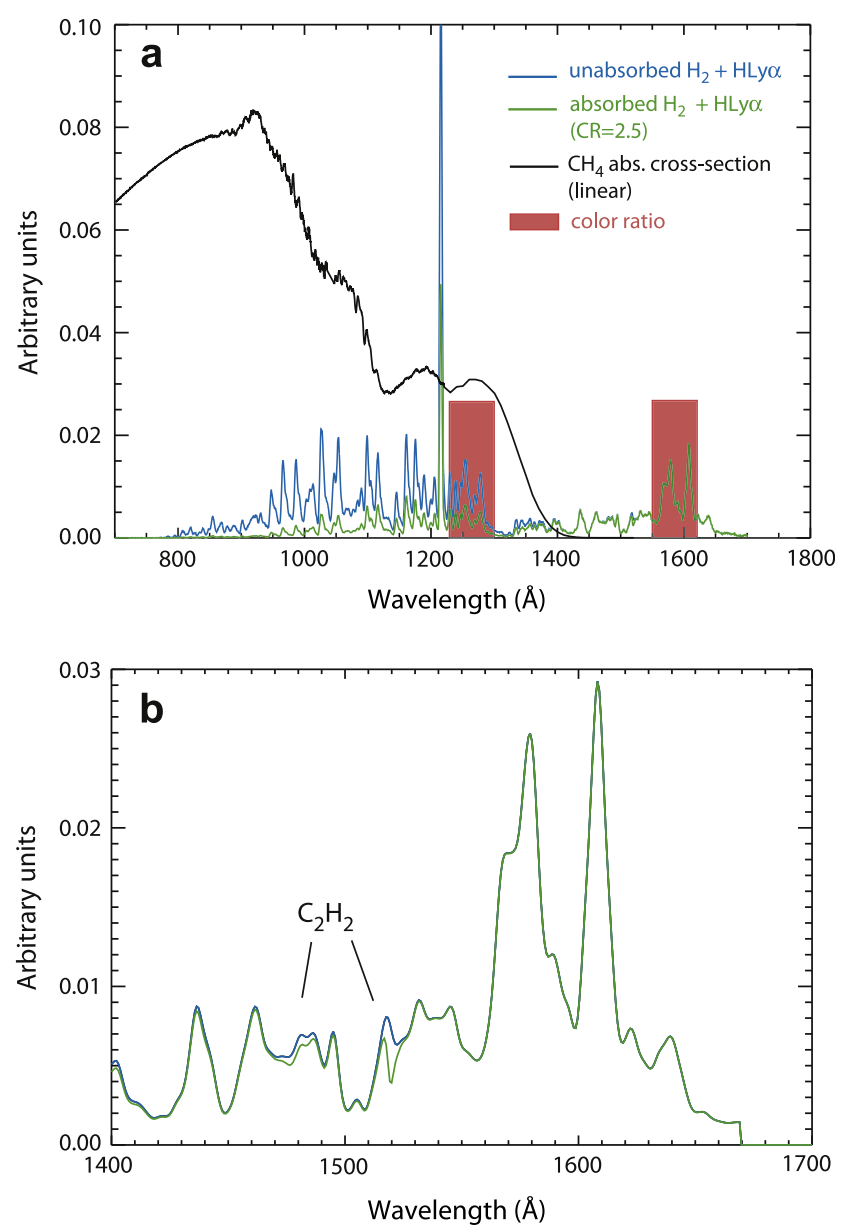

Fig. 4. (a) Laboratory spectrum obtained from impact of $100 \mathrm{eV}$ electrons on $\mathrm{H}_{2}$ gas at $300 \mathrm{~K} \mathrm{[15]} \mathrm{simulating} \mathrm{intrinsic} \mathrm{auroral} \mathrm{emission} \mathrm{(blue} \mathrm{curve).} \mathrm{This} \mathrm{emission} \mathrm{is}$ affected by the hydrocarbon layer (mainly methane) overlying the emission, resulting in an attenuated observed emission (green curve). The $I(1550-1620 \AA$ ) to I(1230-1300 A) ratio (red boxes) defines the FUV color ratio CR and is a measure of the absorption. This example corresponds to a $\mathrm{CR}$ of 2.5 (b) Unabsorbed $\mathrm{H}_{2}$ spectrum (blue) and attenuated $\mathrm{H}_{2}$ spectrum in the 1400-1700 $\AA$ window (green). While the absorbed spectrum in Fig. 4a corresponds to a pressure level of $2 \mu \mathrm{bar}$ in the Jovian atmosphere following the model of Moses et al. [45], absorbed spectrum in Fig. $4 \mathrm{~b}$ has been obtained at $3 \mu$ bar in order to enhance acetylene absorption and clearly illustrates its effects on the auroral emission. (For interpretation of the references to color in this figure legend, the reader is referred to the web version of this article.)

be taken into account to convert observed slant column to vertical values.

The methods described above implicitly assume that the altitude of the hydrocarbon layer is fixed, i.e. that the hydrocarbon column $-\langle E\rangle$ relationship is determined once for all. In reality, this link is influenced by seasonal or local effects which may cause upwelling or downwelling of the hydrocarbon layer. These perturbations are usually put aside in the analysis, as it is currently impossible to monitor the atmospheric structure in real time. Still, the hydrocarbon altitude modifications can be simulated via variations of the eddy diffusion coefficient $\mathrm{K}_{\mathrm{H}}$, which controls the turbulent diffusion and sets the boundary between turbulent and molecular regimes, i.e. the homopause level. This was discussed by Gérard et al. [18], who calculated the CR- $\langle E\rangle$ relationship for different values of $\mathrm{K}_{\mathrm{H}}$. As an example, they found that an observed CR of 4 leads to $\langle E\rangle$ of 100,80 and $50 \mathrm{keV}$ for $\mathrm{K}_{\mathrm{H}}, 2 \times \mathrm{K}_{\mathrm{H}}$ and $10 \times \mathrm{K}_{\mathrm{H}}$, respectively.

Apart from hydrocarbon absorption, another interaction between the auroral photons and the atmosphere is used to situate the auroral peak level: self-absorption. Below $1200 \AA$, photons from transitions connecting to the $v^{\prime \prime}=0,1,2$ level of the $\mathrm{X}^{1} \Sigma_{\mathrm{g}}^{+}$ ground state may be reabsorbed by the overlying $\mathrm{H}_{2}$ (in the temperature range of giant planets stratosphere, $99.99 \%$ of the molecules are in the $v^{\prime \prime}=0,1$ and 2 levels of $\mathrm{X}$ ). The radiative deexcitation that follows produces photons with an energy equal or smaller than the initial absorbed photon. The result is a change in the intensity distribution of the rotational lines, with short wavelength energies partly redistributed to the longer wavelength part of the auroral spectrum. By comparing observed and modeled spectra including self-absorption, the $\mathrm{H}_{2}$ column overlying the aurora and hence $\langle E\rangle$ may be estimated. The advantage of this technique is the direct determination of the $\mathrm{H}_{2}$ column, without the need for any atmospheric model. Two parameters control the amount of self-absorption: the number of self-absorbing molecules (i.e. the $\mathrm{H}_{2}$ column) and their rotational population, which depends on the temperature. In other words, several $\mathrm{H}_{2}$ column-temperature pairs provide very similar self-absorbed spectra, which cannot be discriminated when compared to observed low resolution spectra. It is thus necessary to accurately determine the temperature of the spectrum to fix value of the $\mathrm{H}_{2}$ column. This can only be achieved with spectra that resolve the rotational lines, i.e. spectra with resolution better than $\sim 1 \AA$. These restrictive conditions (spectra in the EUV and high spectral resolution) are rarely met together. The only recent spectra that allowed the determination of $\langle E\rangle$ through self-absorption were obtained by the Far Ultraviolet Spectroscopic Explorer (FUSE) and analyzed by Gustin et al. [27] for Jupiter and Gustin et al. [30] for Saturn.

In addition to its response to precipitating particles creating aurorae, the atmosphere also responds to solar input in the form of an airglow layer on the dayside. In giant planets, this type of emission results from the combination of $\mathrm{H}_{2}$ excitation by photoelectrons produced by solar photons $<500 \AA \AA$ and solar fluorescence due to absorption of solar flux between 900 and $1100 \AA$ ([42,31]). It is still essential to take the effect of hydrocarbon absorption into account to derive the altitude of the emission and the intrinsic brightness, but the processes involved in the airglow phenomenon do not allow to derive the characteristics of the excitation mechanisms from the method used for aurorae.

\section{Applications}

Ultraviolet observations are obtained from spaceborne instruments in order to avoid absorption by $\mathrm{O}_{2}$ in the Earth's atmosphere. Most advances on giant planet's aurora have been obtained from the Voyager Ultraviolet Spectrometer (UVS), the Goddard High Resolution Spectrograph (GHRS), the Space Telescope Imaging Spectrograph (STIS) and the Advanced Camera for Survey (ACS) onboard the Hubble Space Telescope (HST), the Hopkins Ultraviolet Telescope (HUT) on the Space Shuttle, the International Ultraviolet Explorer (IUE) satellite, the Galileo UVS and the Ultraviolet Imaging Spectrograph (UVIS) on board Cassini-Huygens. We focus here on the most recent results obtained from spectroscopic observations involving hydrocarbon measurements.

\subsection{Jupiter}

Jupiter has, by far, the most energetic and brightest aurora of the solar system, a hundred times more energetic than Earth's. The principal source of plasma in Jupiter's environment is Io. The volcanoes on Io eject about 1 ton per sec of sulfur and sulfur dioxide. Part of this material ( $50 \%$, Dols et al. [14]) is ionized and trapped in Jupiter's magnetic field. The charged particles co-rotate with the magnetic field and accumulate along Io's orbit, forming a plasma torus. Part of the plasma diffuses outward in the equatorial 
plane, forming the plasma sheet, partially corotating with Jupiter's magnetic field as well. Jovian's aurora is portrayed by three principal regions of emission (Clarke et al. [9] and references therein). The first is the main oval, characterized by a bright arc extending from dawn to noon, while more diffuse and weaker in the afternoon sector. It is the result of a magnetosphere-ionosphere coupling, associated with the breakdown of plasma corotation in the middle magnetosphere. Second, diffuse emissions are observed in the polar cap (inside the main oval), generally weaker than the main oval, with rapid and sometimes considerable brightening on time scales of minutes. Third, spots at the footprints of Io, Europa and Ganymede are observed, resulting from the electromagnetic interaction between these satellites with Jupiter's magnetic field.

As previously explained, the estimation of the hydrocarbon column density is used to determine the altitude of the auroral emission and the characteristics of the precipitating particles. One of the first models combining $\mathrm{H} 2$ spectra, atmospheric structure and auroral VER was computed by Gladstone and Skinner [22], who compared their model with IUE auroral spectra. They demonstrated that such a model could be used to fit the observations with high accuracy in order to derive the main auroral characteristics. A multi-layer model coupling an electron energy degradation code with a synthetic spectrum generator was used by Dols et al. [13] to simulate emerging auroral UV spectra. A Maxwellian distribution of the electron flux is assumed to precipitate in the atmosphere and several characteristic energies are tested and compared to six spectra observed with GHRS at $\sim 5 \AA$ resolution in the $1200-1700 \AA$ spectral window, which is largely sufficient to provide a good estimate of the hydrocarbon absorption. The initial atmosphere providing $\mathrm{H}, \mathrm{H} 2$, $\mathrm{He}$ and $\mathrm{CH} 4$ density profiles is based on the low latitude model of Gladstone et al. [23]. It was found that $\langle E\rangle$ for the Maxwellian distributions varied from 34 to $80 \mathrm{keV}$, corresponding to altitudes between 226 and $260 \mathrm{~km}$, with acetylene and ethane abundances [0.02-0.2] and [0-0.5] that of methane, respectively. The methane density profile was assumed fixed in order to constrain the electron distribution while the ethane and acetylene relative abundances were scaled from their initial values in order to fine tune the best modeled spectra. This multi-layer model was subsequently improved by a self-consistent calculation of the local temperature and hydrocarbon density perturbations resulting from the auroral precipitation [25]. This last version of the model was used to fit three high resolution EUV spectra obtained with FUSE and a HUT EUV + FUV low resolution auroral spectrum ([27]a). Since the FUSE data resolve the rotational lines and exhibit self-absorbed transitions, the observational constraints imposed on the primary electron distribution include the altitude of the emission (i.e. the $\mathrm{H}_{2}$ column density) and the temperature of the gas at the same time. The initial electron distribution that best fits the data is composed of six Maxwellian distributions. The addition of several distributions allowed to easily modify the shape of the energy spectrum of the primary electrons. In brief, the high energy Maxwellians $(\langle E\rangle$ between 80 and $200 \mathrm{keV}$ ) controlled the altitude of the auroral peak (hence the absorption) while the lower energy components enhanced the temperature gradient above the methane homopause to form a temperature profile consistent with the observed $\mathrm{H}_{2}$ vibrational population.

The $C R$ measured from observed spectra provides a quick estimate of the hydrocarbon absorption without doing a full fit of the data. This is thus an ideal tool to study large datasets. Observations of the Jovian aurora have been made since 1997 with the HST/STIS instrument and provided more than 60 spectra in the $1150-1700 \AA$ range at $\sim 4.8$ and $\sim 12 \AA$ spectral resolution with the $52 \times 0.2$ and $52 \times 0.5 \operatorname{arcsec}^{2}$ slit, respectively. This long slit configuration provides spatially resolved spectra, with a wavelength scale in the dispersion direction and a uniform sam- pling in the spatial direction, which allows a simultaneous study of different auroral regions. Furthermore, several STIS spectra were obtained in the "time-tag" mode, where each recorded photon is kept as a separate event while keeping its wavelength position. It allowed Gérard et al. [18] to examine the time variations of the energy input for different auroral regions. They concluded that the main oval is fairly steady, while emissions inside the oval may exhibit brightening of $50-100 \mathrm{~s}$. The mean energies determined during these polar brightenings are in range of the values found for the main oval and do not show a correlation with the intensity (i.e. energy flux), suggesting that these transient events are due to an increase of the electron flux and not to a hardening of the electron energy. Further investigations by Gustin et al. [28] revealed that the slow and continuous intensity variations in the main oval are correlated with the hydrocarbon absorption hence the energy of the precipitating electrons. They used the CR method to examine the tens of STIS spectra and derive $\langle E\rangle$. Assuming that a brightness of $10 \mathrm{kR}$ (The Rayleigh is a unit for radiance often used by atmospheric scientists: $1 R=10^{6} / 4 \pi$ photons $\mathrm{cm}^{-2} \mathrm{~s}^{-1} \mathrm{sr}^{-1}$ ) in the Lyman and Werner bands is produced by an electron energy flux of $1 \mathrm{~mW} \mathrm{~m}^{-2}$ (e.g. Gérard and Singh [16] or Grodent et al. [25]), the energy flux and $\langle E\rangle$ relationship derived from the main oval spectra were found be compatible with the Knight's theory of field aligned current ([37]), which relates the ionospheric current to the acceleration electric potential experienced by the precipitating electrons along the field lines. Following this hypothesis, data revealed that the magnetospheric electrons in the equatorial plane that are accelerated to produce the aurora are characterized by an energy of $\sim 2.5 \mathrm{keV}$ and a density of $\sim 0.003 \mathrm{~cm}^{-3}$, within the range of values observed during the Voyager flybys. The main auroral oval temporal variations were then interpreted as slow changes in the 'source' electron parameters in the presence of near-steady magnetosphere-ionosphere coupling currents. By contrast, this theory was not validated for the emissions poleward of the main oval, characterized by electron energies similar to those of the main oval, but with smaller energy fluxes, which is indicative of other acceleration processes, such as magnetic reconnections. Overall, these STIS spectra were characterized by $\langle E\rangle$ between 30 and $200 \mathrm{keV}$ and brightnesses between 30 and $510 \mathrm{kR}$. In addition to these regular cases, the STIS dataset includes two exceptionally bright morning arcs observed on 21 September 1999. The maximum brightness peaked at $1.8 \mathrm{MR}$, much more than the values observed in normal conditions The maximum $C R$ reached $\sim 62$, compared to the $\sim 2-10$ values observed generally. The $\mathrm{CR}-\langle E\rangle$ relationship lead to $\langle E\rangle$ of $460 \mathrm{keV}$, which demonstrates the highly variable characteristics of the aurora ([29]).

In addition to the main oval and polar cap emissions, the Io footprint was also examined during these HST campaigns. The CR derived from Io footprint spectra are in the low range $(\sim 1.8$ to $\sim 2.3$ ) compared to the main oval or polar cap $(\sim 1.6$ to $\sim 11)$, with a slow $C R$ decrease with the angular distance from the footprint to the downstream auroral tail. Assuming a Maxwellian electrons distribution, the best models are characterized by variations of $\langle E\rangle$ from $\sim 55 \mathrm{keV}$ at the footprint to $\sim 40 \mathrm{keV} 20^{\circ}$ downstream in the tail [17]. These values contrast with mean energies of $1-2 \mathrm{keV}$ determined by Gérard et al. [20] from the tail vertical profile observed above the disk limb with HST images, peaking at $\sim 900 \mathrm{~km}$ above the 1 bar level. This disagreement is explained by the atmospheric model used by Gérard et al. [17] to situate the altitude of the hydrocarbon layer, which does not take into account the significant influence of the auroral enery input on the atmospheric thermal structure. The lack of appropriate models specifically developed for polar regions, recurrent in auroral studies, will also be invoked in the next section. 


\subsection{Saturn}

Saturn's aurora comprises several components: a main ring emission located at $\sim 75^{\circ}$ of latitude, with a brightness from 1 to several tens of kR. Occasionally, the ring expands, contracts, or evolves to a spiral shape. Auroral rings can either be formed by field-aligned currents, associated with the breakdown of corotation of the plasma rotating in the middle magnetosphere (as in the case of Jupiter), or by interactions between the solar wind and the planet's magnetosphere at the open-closed field line boundary (as for the Earth). Theoretical and observational studies indicate that the latter is more likely in Saturn's case (e.g. Kurth et al. [38] and references therein for more informations). Other types of emissions have been observed (transient isolated elongated spots [50], equatorward faint emission [26], multiple arcs (Radioti et al. [50]), etc...., which makes Saturn's UV aurora a very rich and fascinating phenomenon. While the overall brightness of the main ring responds rapidly to the solar wind dynamic pressure variations (e.g. Clarke et al. [10]), considerable longitudinal structure and time variations over interval of a few hours are also observed, despite the absence of observable external triggers and solar wind activations (e.g. Gérard et al. [19]), which suggests that the aurora is also influenced by an intrinsically dynamical magnetosphere. Saturn's aurora can thus be depicted as an intermediate case between a Solar controlled aurora (like Earth) and a internally triggered aurora (like Jupiter).

Spectroscopic investigations of FUV Saturn aurorae have been principally done with HST/STIS and Cassini/UVIS instruments. Unlike Jupiter, Saturn's spectra exhibit a weak absorption, when present: Voyager spectra obtained in 1980 did not show methane attenuation except in one case ([52]). Six STIS spectra obtained in 2000 did show a weak attenuation by methane, with CR between 1.3 and 1.5, without signature of ethane and acetylene [19]. Although models of Saturn's atmosphere exist (e.g. Moses et al. [45] or Ollivier et al. [48]), a model combining a spectral generator with an atmosphere self-consistently responding to the electron precipitation input at auroral latitudes is not available for Saturn. The two layers model consisting of an auroral emitting layer and an absorbing hydrocarbon layer is usually used instead to determine the energy of the primary electrons. A synthetic $\mathrm{H}_{2}$ spectrum at a rovibrational temperature of $400 \mathrm{~K}$ (assumed value at the altitude of aurora) was used by Gérard et al. [19] to fit the STIS spectra from 2000. They needed vertical $\mathrm{CH}_{4}$ columns from $4 \times 10^{15}$ to $1.2 \times 10^{16} \mathrm{~cm}^{-2}$ to best fit the observations, which corresponds to vertical $\mathrm{H}_{2}$ columns between 5 and $8 \times 10^{19} \mathrm{~cm}^{-2}$ in the atmospheric model of Moses et al. [45]. A table of stopping power relating the penetration depth of mono-energetic electrons into $\mathrm{H}_{2}$ was used to determine $\langle E\rangle$, and values between 13 and $17 \mathrm{keV}$ were found, much less than the typical 30-200 keV determined for Jupiter [28]. These lower energies are associated with pressure levels between 0.15 and $0.3 \mu$ bars, in agreement with the $0.1-0.2 \mu$ bar range determined from FUSE EUV spectra using self-absorption ([30]).

The Cassini mission to Saturn brought new perspective to auroral studies, because of its regular monitoring of the dayside and nightside aurora, closeness and viewing geometry which includes full nadir views of the aurora. UVIS has a FUV channel from 1115 to $1912 \AA$, usually associated with the low resolution slit, which provides 64 spatial pixels of $1 \mathrm{mrad}$ along the slit by $1.5 \mathrm{mrad}$ across the slit, i.e. a detector resolution of 64 pixels in the spatial direction and 1024 pixels in the dispersion direction. This configuration and continuous slew of the spacecraft scanning the aurora provides images with full spectral information for each pixel. The few published cases confirm the STIS findings, i.e. brightnesses from a few $\mathrm{kR}$ to a few tens of $\mathrm{kR}$ and moderate $\mathrm{CH}_{4}$ absorption. More than 500 spectra have been extracted for UVIS images and are currently under study. Preliminary results confirm that absorption is occasional at Saturn, with methane signature in $\sim 20 \%$ of the observations (Gustin et al., [59]). The large number of spectra from different regions of the aurora will allow to determine the energy of the electrons and study the dependence of the emission to local time, latitude, etc.,... and see if different regions can be associated with different acceleration processes. The weak and occasional attenuation of the aurora suggests that the altitude of the emission is situated near the top of the methane layer, around $650 \mathrm{~km}$ in the Moses et al. [45] model.

An improvement to the two layer model associated with a mono-energetic beam was operated recently by coupling the atmosphere of Moses et al. [45] to a Monte-Carlo electron transport code [21], which calculates the vertical distribution of the $\mathrm{H}_{2}$ auroral emission. The Monte-Carlo method solves the Boltzmann equation, which describes the elastic, inelastic and ionizing collisions of the precipitating electrons with $\mathrm{H}_{2}, \mathrm{H}$ and He atoms in the ambient gas, as well as the creation of the secondary electrons. It provides the volume emission rate profile for the $\mathrm{H}_{2} \mathrm{Ly}-$ man and Werner bands within the initial atmosphere (not evolving in this approach). The model is divided into 49 layers and the methane column overlying each layer is applied to the unabsorbed laboratory spectrum. The emergent spectrum, sum of the individual spectra weighted by the VER of each layer, is compared to the observations. This model has been applied to STIS and UVIS FUV spectra obtained in March and April 2011 [21]. Several single Maxwellian distribution were tested, and distributions with mean energies from less than 3 to $10 \mathrm{keV}$ best matched the observations, corresponding to an auroral emission peaking in the 610-650 km range in the atmosphere of Moses et al. [45].

On the other hand, images obtained with HST- brought out the auroral emission profile in a limb viewing geometry. Analysis of these images by Gérard et al. [20] situate the auroral peak between 900 and $1300 \mathrm{~km}$ above the 1 bar level, i.e. significantly higher than what derived from spectroscopic observations. This discrepancy puts forward a weakness of the methane-based method used to derive auroral characteristics: the lack of atmospheric model appropriate for auroral latitudes.

For both Jupiter and Saturn, only low latitude models have been developed. The decrease of gravity, weaker solar contribution and very localized energy input due to auroral electrons makes highlatitude atmospheres probably significantly different from low-latitude ones, in terms of structure of the atmosphere, constituent abundance and temperature profiles. Development of high latitude models, notably based on stellar and solar occultations, are part of the work in progress allowing a continuous improvement in the understanding of aurora on giant planets.

As for Jupiter, Saturn exhibits a spot due to the electrodynamic coupling between Enceladus and Saturn, like that which links Jupiter with Io, Europa and Ganymede. The Enceladus footprint, fed by particle originating from its cryovolcanic activity, has been detected recently with the UVIS spectrograph [49]. The several observed spectra of the Enceladus spot did not exhibit methane absorption, suggesting precipitation of particles with energies less than $10 \mathrm{keV}$.

\section{Summary}

The aurora on giant planets is the result of inelastic collision between magnetospheric electrons with $\mathrm{H}_{2}$ molecules, which de-excite through vibronic transitions and produce emission bands in the UV spectral window. This auroral layer forms in the vicinity of an hydrocarbon layer, which potentially attenuates the observed aurora in specific UV wavelengths. Determination of the amount of absorption, mainly due to methane, allows to locate the altitude of 
the aurora. Assuming that the latter is directly linked to the mean energy $\langle E\rangle$ of the precipitating electrons, this hydrocarbon absorption is an essential tool used to determine the characteristics of giant planet's aurora, such as its intrinsic brightness, altitude of emission and $\langle E\rangle$, which constrains models describing the magnetosphere-ionosphere interaction and the acceleration processes involved.

Three concepts are used to relate methane absorption with $\langle E\rangle$. The first one considers a emitting layer overlaid by an absorbing layer and excited by mono-energetic electrons. Although very simple, this model provides consistent estimates of the auroral characteristics. The second, more realistic concept is based on a multilayered atmosphere, excited by an energy spectrum of primary electrons, usually a Maxwellian distribution. Each layer is associated with an auroral spectrum and the emergent spectrum, sum of spectra from each layer, is compared to observed spectra to determine the auroral characteristics. For both Jupiter and Saturn, a low latitude atmospheric model is used to locate the emission. While the temperature and methane profiles self-consistently adjust to the auroral input in the case of Jovian studies, the multi-layered model considers a steady atmosphere in the case of Saturn and adjustments to the auroral input is a next step required in future studies. A third concept uses the ratio between an unabsorbed portion (1550-1620 $\AA$ ) and a absorbed portion (1230-1300 $\AA$ ) of the FUV spectrum. This color ratio thus relates the hydrocarbon absorption to the altitude of the emission, i.e. the energy of the precipitating particles. Although oversimplified, this approach is ideal to examine large datasets and study temporal variations of auroral characteristics.
Table 1 summarizes the auroral characteristics determined from these methods for both Jupiter and Saturn. UV aurorae at Jupiter are at least one order of magnitude brighter than Saturn's case. The energy of the precipitating electrons is also one order of magnitude larger in the Jovian case, which sets the level of the auroral emission near the $1 \mu$ bar level, much deeper than the $\sim 0.1 \mu$ bar observed at Saturn. Example spectra presented in Table 1 reveal that FUV auroral emission is composed of the atomic hydrogen Lyman alpha line and $\mathrm{H}_{2}$ rovibronic bands, attenuated by hydrocarbons. Because of the larger number of instruments active in the FUV bandwidth and the methane absorption cross-section which absorbs lower part of the FUV while leaving the longer part unattenuated, ideal to determine the number of absorbing molecules, FUV spectra are well suited to determine the number of absorbing molecules and thus broadly used in this field of study.

For both planets, only low-latitude models are available to derive the auroral characteristics. In the future, more appropriate high-latitude models with adapted gravity and including processes like auroral energy input, heating and transport mechanisms should be derived from various observations such as stellar or solar occultations to determine auroral characteristics with the highest possible degree of accuracy.

The NASA JUNO mission to Jupiter (launched on August 2011) and the ESA's Jupiter Icy Moon Explorer (JUICE) mission (to be lauched in 2022) are the latest projects that will bring new insights on the auroral phenomenon. In particular, the polar orbits described by JUNO and the observations of the UV aurora with simultaneous measurements of the energy of the precipitating particle will bring strong constraints on the models produced so far. In the near

Table 1

Summary of auroral characteristics deduced from hydrocarbon absorption.

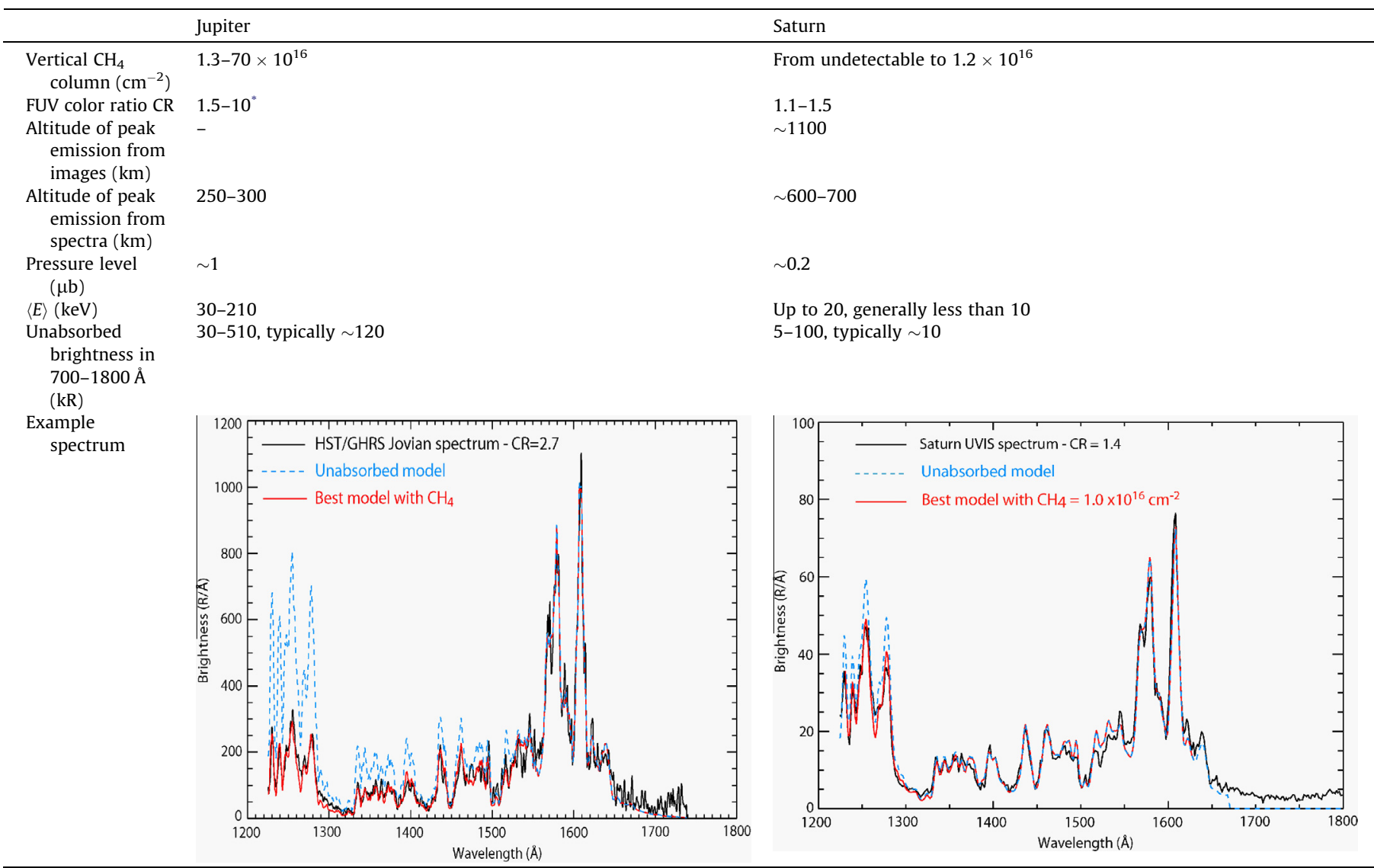

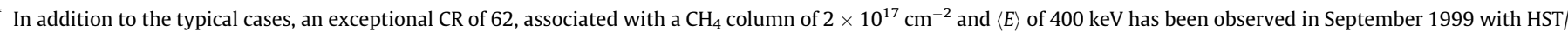
STIS (see Gustin et al. [29]). 
future, the scientific principle described in this study could be extended to Uranus. In addition to HST observations, a huge step forward would be reached if a mission like Uranus Pathfinder ([2]) is developed. A better knowledge of the "Ice Giants" Uranus and Neptune would fill the gap in our understanding of the Solar System formation and evolution, and unravel numerous physical and chemical processes that need to be apprehended to have a better picture of our neighborhood.

\section{Acknowledgments}

Financial support to US participants was provided by NASA and the Cassini Project. J.-C.G. and A.R. acknowledge support from the Belgian Fund for Scientific Research (FNRS). The PRODEX program of ESA and the University of Liège provided financial support for this research.

\section{References}

[1] J.W. Au, G. Cooper, G.R. Burton, T.N. Olney, C.E. Brion, Chem. Phys. 173 (1993) 209-239.

[2] C.S. Arridge, and 78 colleagues, Exp. Astron, 33 (2012): 753-791 doi: 10.1007/ s10686-011-9251-4.

[3] A. Bhardwaj, G.R. Gladstone, Rev. Geoph. 38 (2000) 295-353, http://dx.doi.org/ 10.1029/1998RG000046.

[4] G. Branduardi-Raymont, A. Bhardwaj, R.F. Elsner, G.R. Gladstone, G. Ramsay, P. Rodriguez, R. Soria, J.H. Waite Jr., T.E. Cravens, Astron. Astrophys. 463 (2007) 761, http://dx.doi.org/10.1051/0004-6361:20066406.

[5] A.L. Broadfoot, and 16 colleagues, Science 204 (1979) 979-982.

[6] A.L. Broadfoot, B.R. Sandel, D.E. Shemansky, J.B. Holberg, G.R. Smith, D.F. Strobel, J.C. McConnell, S. Kumar, D.M. Hunten, S.K. Atreya, T.M. Donahue, H.W. Moos, J.L. Bertaux, J.E. Blamont, R.B. Pomphrey, S. Linik, Science 212 (1981) 206-211.

[7] A.L. Broadfoot, and 18 colleague, Science 233 (1986) 74-79 doi: 10.1126/ science.233.4759.74.

[8] F.Z. Chen, C.Y.R. Wu, J. Quant. Spectrosc. Radiat. Transfer 85 (2004) 195-209.

[9] J.T. Clarke, D. Grodent, S.W.H. Cowley, E.J. Bunce, P. Zarka, J.E.P. Connerney, T. Satoh, Jupiter's Aurora, Jupiter. The Planet, Satellites and Magnetosphere, vol. 1, Cambridge Planetary Science, ISBN 0-521-81808-7, 2004 (639-670).

[10] J.T. Clarke, 20 colleagues, J. Geophys. Res. 114 (2009) A05210, http:// dx.doi.org/10.1029/2008JA013694.

[11] G. Cooper, G.R. Burton, C.E. Brion, J. Electron Spectrosc. Relat. Phenom. 73 (2) (1995) 139-148.

[12] R.W. Ditchburn, Proc. Roy. Soc. Lond. A 229 (1955) 44-62.

[13] V. Dols, J.-C. Gérard, J.T. Clarke, J. Gustin, D. Grodent, Icarus 147 (2000) 251266.

[14] V. Dols, P.A. Delamere, F. Bagenal, J. Geophys. Res. 113 (2008) A09208, http:// dx.doi.org/10.1029/2007JA012805.

[15] D. Dziczek, J.M. Ajello, G.K. James, D.L. Hansen, Phys. Rev. A 61 (2000) 647021-64702-4.

[16] J.-C. Gérard, V. Singh, J. Geophys. Res. 87 (1982) 4525-4532, http://dx.doi.org/ 10.1029/JA087iA06p04525.

[17] J.-C. Gérard, J. Gustin, D. Grodent, P. Delamere, J.T. Clarke, J. Geophys. Res. 107 (A11) (2002) 1394, http://dx.doi.org/10.1029/2002JA009410.

[18] J.-C. Gérard, J. Gustin, D. Grodent, J.T. Clarke, J. Geophys. Res. 108 (A8) (2003) 1319, http://dx.doi.org/10.1029/2003JA009901.

[19] J.-C. Gérard et al., J. Geophys. Res. 111 (2006) A12210, http://dx.doi.org/ 10.1029/2006JA011965.

[20] J.C. Gérard, B. Bonfond, J. Gustin, D. Grodent, J.T. Clarke, D. Bisikalo, V. Shematovich, Geophys. Res. Lett. 36 (2009) L02202, http://dx.doi.org/10.1029/ 2008 GL036554.

[21] J.-C. Gérard, J. Gustin, W.R. Pryor, D. Grodent, B. Bonfond, A. Radioti, G.R. Gladstone, J.T. Clarke, J.D. Nichols, Icarus 223 (1) (2013) 211-221. http:// dx.doi.org/10.1016/j.icarus.2012.11.033.

[22] G.R. Gladstone, T.E. Skinner, Spectral Analysis of Jovian Auroral Emissions, NASA Special Publication Series, NASA-SP-494, 1989 (pp. 221-228).

[23] G.R. Gladstone, M. Allen, Y.L. Yung, Icarus 119 (1996) 1-52.

[24] D. Grodent, G.R. Gladstone, J.-C. Gérard, V. Dols, J.H. Waite, Icarus 128 (1997) 306-321.

[25] D. Grodent, J.H. Waite Jr., J.C. Gérard, J. Geophys. Res. 106 (2001) 1293312952.
[26] D. Grodent, A. Radioti, B. Bonfond, J.-C. Gérard, J. Geophys. Res. 115 (2010) A08219, http://dx.doi.org/10.1029/2009JA014901.

[27] J. Gustin, P.D. Feldman, J.-C. Gérard, D. Grodent, A. Vidal-Madjar, L. Ben Jaffel, J.-M. Desert, H.W. Moos, D.J. Sahnow, H.A. Weaver, B.C. Wolven, J.M. Ajello, J.H. Waite, E. Roueff, H. Abgrall, Icarus 171 (2004) 336-355.

[28] J. Gustin, J.-C. Gérard, D. Grodent, S.W.H. Cowley, J.T. Clarke, A. Grard, J Geophys. Res. 109 (2004) A10205, http://dx.doi.org/10.1029/2003JA010365.

[29] J. Gustin, S.W.H. Cowley, J.-C. Gérard, G.R. Gladstone, D. Grodent, J.T. Clarke, J. Geophys. Res. 111 (2006) A09220, http://dx.doi.org/10.1029/2006JA011730. $13 \mathrm{p}$.

[30] J. Gustin, J.-C. Gérard, W. Pryor, P.D. Feldman, D. Grodent, G. Holsclaw, Icarus 200 (2009) 176-187.

[31] J. Gustin, I. Stewart, J.-C. Gérard, L. Esposito, Icarus 210 (2010) 270-283, http:// dx.doi.org/10.1016/j. icarus.2010.06.031.

[32] J. Gustin, B. Bonfond, D. Grodent, J.-C. Gérard, J. Geophys. Res. 117 (2012) A07316, http://dx.doi.org/10.1029/2012JA017607.

[33] Y. Hui, D.R. Schultz, V.A. Kharchenko, A. Bhardwaj, G. Branduardi-Raymont, P.C. Stancil, T.E. Cravens, C.M. Lisse, A. Dalgarno, J. Geophys. Res. 115, A07102 doi: $10.1029 / 2009 J A 014854$.

[34] K. Kameta, N. Kouchi, M. Ukai, Y. Hatano, J. Electron Spectrosc. Related Phenom. 123 (2002) 225-238.

[35] K. Kameta, S. Machida, M. Kitajama, M. Ukai, N. Kouchi, Y. Hatano, K. Ito, J. Electron Spectrosc. Related Phenom. 79 (1996) 391-393.

[36] V. Kharchenko, A. Bhardwaj, A. Dalgarno, D.R. Schultz, P.C. Stancil, J. Geophys. Res. 113, A08229 doi: 10.1029/2008JA013062.

[37] S. Knight, Planet. Space Sci. 21 (1973) 741.

[38] W.S. Kurth, E.J. Bunce, J.T. Clarke, F.J. Crary, D.C. Grodent, A.P. Ingersoll, U.A Dydudina, L. Lamy, D.G. Mitchell, A.M. Persoon, W.R. Pryor, J. Saur, T. Stallard, Auroral processes, Saturn from Cassini-Huygens, ISBN 978-1-4020-9216-9, 2009, pp. 333-350.

[39] A.H. Laufer, J.R. McNesby, Can. J. Chem. 43 (1965) 3487-3490.

[40] L. Lamy, and 18 colleagues, Geophys. Res. Lett., 39, L07105 doi: 10.1029/ 2012GL051312, 2012.

[41] A.Y.T. Lee, Y.L. Yung, B.M. Cheng, M. Bahou, C.-Y. Chung, Y.P. Lee, Astrophys. J. 551 (2001) L93-L96.

[42] W. Liu, A. Dalgarno, Astrophys. J. 462 (1996) 502-518.

[43] W. Liu, D.R. Schultz, Astrophys. J. 530 (2000) 500-503, http://dx.doi.org/ $10.1086 / 308367$

[44] T.A. Livengood, D.F. Strobel, H.W. Moos, J. Geophys. Res. 95 (1990) $10375-$ 10388, http://dx.doi.org/10.1029/JA095iA07p10375.

[45] J.I. Moses, T. Fouchet, B. Bézard, G.R. Gladstone, E. Lellouch, H. Feuchtgruber, J. Geophys. Res. 110 (2005) E08001, http://dx.doi.org/10.1029/2005JE002411.

[46] T. Nakayama, K. Watanabe, J. Chem. Phys. 40 (1964) 558-561.

[47] N.F. Ness, M.H. Acuna, K.W. Behannon, L.F. Burlaga, J.E.P. Connerney, R.P. Lepping, Science 233 (1986) 85-89.

[48] J.-L. Ollivier, F. Billebaud, P. Drossart, M. Dobrijévic, M. Roos-Serote, T. AugustBernex, I. Vauglin, Astron. Astrophys. 356 (2000) 347-356.

[49] W.R. Pryor, 28 authors, Nature 472 (2011) 331-333, http://dx.doi.org/10.1038/ nature09928.

[50] A. Radioti, D. Grodent, J.-C. Gérard, E. Roussos, C. Paranicas, B. Bonfond, D.G. Mitchell, N. Krupp, S. Krimigis, J.T. Clarke, J. Geophys. Res. 114 (2009) A3, http://dx.doi.org/10.1029/2008JA013632.

[51] D. Rego, R. Prangé, L. Ben Jaffe, J. Geophys. Res. 104 (E3) (1999) 5939-5954 http://dx.doi.org/10.1029/1998JE900048.

[52] B.R. Sandel, D.E. Shemansky, A.L. Broadfoot, J.B. Holberg, G.R. Smith, Science 215 (1982) 548-553.

[53] L.M. Trafton, V. Dols, J.-C. Gérard, J.H. Waite, G.R. Gladstone, G. Munhoven, Astrophys. J. 507 (1998) 955-967, http://dx.doi.org/10.1086/306338.

[54] A.R. Vasavada, A.H. Bouchez, A.P. Ingersoll, B. Little, C.D. Anger, and the Galileo SSI Team, J. Geophys. Res. 104(E11) (1999) 27133-27142 doi: 10.1029/ 1999JE001055.

[55] J.H. Waite Jr., F. Bagenal, F. Seward, C. Na, G.R. Gladstone, T.E. Cravens, K.C. Hurley, J.T. Clarke, R. Elsner, S.A. Stern, J. Geophys. Res. 99 (A8) (1994) 1479914809, http://dx.doi.org/10.1029/94JA01005.

[56] C.Y.R. Wu, F.Z. Chen, D.L. Judge, J. Geophys. Res. 106 (2001) 7629-7636.

[57] Y.L. Yung, G.R. Gladstone, K.M. Chang, J.M. Ajello, S.K. Srivastava, Astrophys. J. 254 (1982) L65-L69, http://dx.doi.org/10.1086/183757.

[58] A.L. Broadfoot, S.K. Atreya, J.L. Bertaux, J.E. Blamont, A.J. Dessler, T.M. Donahue W.T. Forrester, D.T. Hall, F. Herbert, J.B. Holberg, D.M. Hunten, V.A. Krasnopolsky, S. Linick, J.I. Lunine, J.C. Mcconnell, H.W. Moos, B.R. Sandel, N.M. Schneider, D.E. Shemansky, G.R. Smith, D.F. Strobel, R.V. Yelle, Science 246 (1989) 1459-1466.

[59] J. Gustin, J.-C. Gérard, D. Grodent, A. Radioti and W. Pryor, UVIS FUV spectra of Saturn's aurora, Europlanet workshop, Liege, Belgium, 18-20 October, 2010. 